\title{
Control of the Nigrostriatal Dopamine Neuron Activity and Motor Function by the Tail of the Ventral Tegmental Area
}

\author{
Romain Bourdy ${ }^{1,2,6}$, María-José Sánchez-Catalán ${ }^{1,6}$, Jennifer Kaufling 1,3, Judith J Balcita-Pedicino4, \\ Marie-José Freund-Mercier ${ }^{1,2}$, Pierre Veinante ${ }^{1,2}$, Susan R Sesack ${ }^{4}$, François Georges ${ }^{3,5,7}$ and \\ Michel Barrot*, ${ }^{*}, 7$ \\ I'Institut des Neurosciences Cellulaires et Intégratives, Centre National de la Recherche Scientifique, Strasbourg, France; '2Université de Strasbourg, \\ Strasbourg, France; ${ }^{3}$ Centre National de la Recherche Scientifique, Interdisciplinary Institute for Neuroscience, Bordeaux, France; ${ }^{4}$ Department of \\ Neuroscience and Department of Psychiatry, University of Pittsburgh, Pittsburgh, Pennsylvania, USA; ${ }^{5}$ Université de Bordeaux, Interdisciplinary \\ Institute for Neuroscience, Bordeaux, France
}

Midbrain dopamine neurons are implicated in various psychiatric and neurological disorders. The GABAergic tail of the ventral tegmental area (tVTA), also named the rostromedial tegmental nucleus (RMTg), displays dense projections to the midbrain and exerts electrophysiological control over dopamine cells of the VTA. However, the influence of the VVTA on the nigrostriatal pathway, from the substantia nigra pars compacta $(\mathrm{SNc})$ to the dorsal striatum, and on related functions remains to be addressed. The present study highlights the role played by the tVTA as a GABA brake for the nigrostriatal system, demonstrating a critical influence over motor functions. Using neuroanatomical approaches with tract tracing and electron microscopy, we reveal the presence of a tVTA-SNc-dorsal striatum pathway. Using in vivo electrophysiology, we prove that the tVTA is a major inhibitory control center for SNc dopamine cells. Using behavioral approaches, we demonstrate that the tVTA controls rotation behavior, motor coordination, and motor skill learning. The motor enhancements observed after ablation of the tVTA are in this regard comparable with the performance-enhancing properties of amphetamine, a drug used in doping. These findings demonstrate that the tVTA is a major GABA brake for nigral dopamine systems and nigrostriatal functions, and they raise important questions about how the tVTA is integrated within the basal ganglia circuitry. They also warrant further research on the tVTA's role in motor and dopamine-related pathological contexts such as Parkinson's disease. Neuropsychopharmacology (20I4) 39, 2788-2798; doi:I0.I038/npp.20I4.129; published online 25 June 2014

\section{INTRODUCTION}

Dopamine regulation of basal ganglia circuitry is critical to fine motor control and to associated pathologies such as Parkinson's disease (Albin et al, 1989; Redgrave et al, 2010). In this circuit, the nigrostriatal pathway from dopamine neurons of the substantia nigra pars compacta $(\mathrm{SNc})$ to the dorsal striatum is controlled by various inhibitory GABAergic afferents (Tepper and Lee, 2007). The striatum, the external globus pallidus, and the substantia nigra pars reticulata are major contributors to this GABAergic control of $\mathrm{SNc}$ dopaminergic activity. Within the midbrain, the tail of the ventral tegmental area (tVTA) (Kaufling et al, 2009, 2010a; Perroti et al, 2005) also named the rostromedial tegmental (RMTg) nucleus (Jhou et al, 2009a, 2009b) appears as a GABAergic inhibitory structure displaying

*Correspondence: Dr M Barrot, Institut des Neurosciences Cellulaires et Intégratives, CNRS UPR32 I2, 5 rue Blaise Pascal, Strasbourg 67084, France. Tel: +33 368 85। 450; Fax: +33 388613 347; E-mail: mbarrot@inci-cnrs.unistra.fr

${ }^{6}$ These authors contributed equally to this work.

${ }^{7}$ These authors contributed equally to this work.

Received 28 February 2014; revised 16 May 2014; accepted 29 May 2014; accepted article preview online 4 June 2014 heavy projections to the VTA and the SNc, suggesting that the tVTA could regulate the activity of dopamine systems (Barrot et al, 2012; Bourdy and Barrot, 2012). In the previous years, this inhibitory control has been investigated in the contexts of VTA responses to drugs of abuse (Jalabert et al, 2011; Jhou et al, 2012, 2013; Lecca et al, 2011, 2012; Matsui and Williams, 2011) and of the habenulo-VTA pathway in avoidance behavior (Stamatakis and Stuber, 2012) and in reward prediction error (Hong et al, 2011), demonstrating that the tVTA is indeed an important GABA brake for VTA dopamine neurons. However, the influence of the tVTA on the nigrostriatal pathway and on motor functions remains to be explored.

In the present study, we combined neuroanatomical, electrophysiological, and behavioral approaches to demonstrate the functional role played by the tVTA as a GABA brake for the nigrostriatal system and motor function. With pathway tract tracing, electron microscopic analysis of synapses and in vivo extracellular single-cell recordings, we demonstrate the inhibitory connectivity between tVTA efferent fibers and SNc dopamine neurons. Moreover, the experimental ablation of tVTA reveals a major influence of this structure on motor skill learning and on motor performance. 


\section{MATERIALS AND METHODS}

\section{Subjects}

Experiments were performed in adult male Sprague-Dawley rats (Janvier, France). The rats were habituated to the facilities for at least a week before starting the procedures, and the animals were 7-9 weeks old (ie, 250-350 g) at surgery time or at testing time when no surgery was involved. They were housed under standard conditions $\left(22^{\circ} \mathrm{C}\right.$, 12 -hour light/ dark cycle). The experiments were in accordance with the CNRS and European community's council directives, with animal facilities and procedures approved by regional veterinary office and with scientists having the legal certificate for experimentation on living animals.

\section{Drugs}

Ibotenic acid (1\% in phosphate-buffered saline (PBS) $0.1 \mathrm{M}$; $0.3 \mu \mathrm{l}$ ) was injected unilaterally into the VTA and unilaterally or bilaterally into the tVTA using Hamilton syringes with 33-gauge needles. Control animals received $0.3 \mu \mathrm{l}$ of PBS. The 6-hydroxydopamine hydrobromide (6-OHDA; $0.25 \%$ in $0.9 \% \mathrm{NaCl}$ with $0.01 \%$ ascorbic acid) was injected unilaterally into the $\mathrm{SNc}(4 \mu \mathrm{l}$ for large lesion or $2 \mu \mathrm{l}$ for partial lesion) or bilaterally into the dorsolateral striatum (2 $\mu \mathrm{l} /$ hemisphere). D-amphetamine sulfate was injected intraperitoneally (i.p., in $0.9 \% \mathrm{NaCl}$ ) at $3 \mathrm{mg} / \mathrm{kg}$ for rotational behavior and $1 \mathrm{mg} / \mathrm{kg}$ for rotarod experiments.

\section{Surgery}

Rats were anesthetized under ketamine/xylazine $(87 \mathrm{mg} / \mathrm{kg}$ and $13 \mathrm{mg} / \mathrm{kg}$ respectively, i.p.) for tract tracing and 6 OHDA surgeries, under isoflurane for electrophysiology (3\% for induction, $2 \%$ for surgery) and under sodium pentobarbital $(50 \mathrm{mg} / \mathrm{kg}$, i.p.) for ibotenic acid lesions. Stereotaxic coordinates relative to bregma were adjusted to the animal weight, coordinates (in $\mathrm{mm}$ ) were as follows (Paxinos and Watson, 2007): tVTA, anteroposterior (AP) $=$ -6.5 , lateral $(\mathrm{L})= \pm 1.5$, ventral $(\mathrm{V})=-7.8, \pm 6^{\circ}$ lateral angle and dorsolateral striatum, $\mathrm{AP}=+1, \mathrm{~L}= \pm 4.3$, $\mathrm{V}=-4, \pm 6^{\circ}$ lateral angle for tract tracing and behavior; tVTA, $\mathrm{AP}=-7, \mathrm{~L}=+0.4, \mathrm{~V}=-7.4$ and $\mathrm{SNc}, \mathrm{AP}=-5$, $\mathrm{L}= \pm 1.5, \mathrm{~V}=-7.5$ for electrophysiology; $\mathrm{SNc}, \mathrm{AP}=-5.2$, $\mathrm{L}= \pm 2.6, \mathrm{~V}=-7.6, \pm 6^{\circ}$ lateral angle and $\mathrm{VTA}, \mathrm{AP}=-5$, $\mathrm{L}= \pm 1.6, \mathrm{~V}=-7.5, \pm 6^{\circ}$ lateral angle for behavior. Verticality was taken from the dura.

\section{Tract Tracing}

Biotinylated dextran amine (BDA; $5 \%$ in $0.25 \mathrm{M}$ potassium acetate), Phaseolus vulgaris leucoagglutinin (PhaL; $2.5 \%$ in $10 \mathrm{mM}$ sodium phosphate buffer) and cholera toxin $\beta$-subunit (CTb; $0.25 \%$ in $0.1 \mathrm{M}$ Tris) were iontophoretically delivered ( +1 to $+6 \mu \mathrm{A}, 7-10 \mathrm{~s}$ on/off cycles for $10-20 \mathrm{~min})$ with glass micropipettes $(10-40 \mu \mathrm{m}$ tip diameter) as previously described (Balcita-Pedicino et al, 2011; Kaufling et al, 2009; Omelchenko et al, 2009).

\section{Histochemistry}

One week following tracer injection or immediately after behavioral testing, rats were anesthetized with sodium pentobarbital and perfused with $100 \mathrm{ml}$ phosphate buffer (0.1 M, pH 7.4) followed by $500 \mathrm{ml}$ of a paraformaldehyde solution ( $4 \%$ in phosphate buffer). Brains were collected, postfixed overnight, and coronal sections $(40 \mu \mathrm{m})$ were cut on a vibratome. Histochemistry for BDA and immunohistochemistry was done as previously described (Kaufling et al, 2009, 2010a; Omelchencko et al, 2009). Primary antibodies were directed against: CTb (Sigma C3062, 1/20000), tyrosine hydroxylase (TH) (Millipore-Chemicon MAB318, 1/2500), or NeuN (Millipore MAB377, 1/5000). Visualization was performed with biotinylated secondary antibodies and peroxidase/DAB reaction after avidin-biotinperoxidase complex amplification (ABC Elite; Vector Laboratories, Burlingame, CA, USA).

\section{Electron Microscopy}

Rats were a subset of those used for the prior study of the tVTA/RMTg projection to the VTA (Balcita-Pedicino et al, 2011). For the present analysis, four animals were chosen as also having good transport of PhaL to the SNc. Ultrathin sections $(60 \mathrm{~nm}$ ) were cut from the core of the SNc (with slight inclusion of the immediately adjacent pars reticulata), stained with heavy metals, and examined by electron microscopy as previously described (Balcita-Pedicino et al, 2011). Primary antibodies were specific for PhaL (Vector AS-2300, 1/1000) and TH (MAB318, 1/5000). PhaL visualization was performed with biotinylated secondary antibodies and peroxidase/DAB reaction after avidinbiotin-peroxidase complex amplification. Then, the sections were processed for TH immunogold-silver staining, using gold-conjugated secondary antibody goat antirabbit (Aurion, Electron Microscopy Science, 1/50, Hatfield, PA, USA) and IntenSE M silver kit reagent (GE Healthcare, Chalfont St Giles, UK) for the silver intensification of gold label. The semiquantitative ultrastructural analysis was done as previously described (Omelchenko et al, 2009; Balcita-Pedicino et al, 2011).

\section{Electrophysiology}

A glass micropipette (2-3 $\mu \mathrm{m}$ tip diameter; $4-6 \mathrm{M} \Omega$ ) filled with $2 \%$ pontamine sky blue in $0.5 \mathrm{M}$ sodium acetate was lowered into the SNc. Dopamine neurons were identified according to their electrophysiological features (Ungless et al, 2004). The extracellular potential was recorded with an Axoclamp2B (Axon Instrument) or Neurodata IR283 (Cygnys Technology; experiment Figure 4b) amplifier in the bridge mode $v s$ a reference electrode in contact with the skull $(\times 10$ gain). The signal was further amplified $(\times 100$ gain) and filtered (low-pass $300 \mathrm{~Hz}$, high-pass $0.5 \mathrm{kHz}$ ) via a differential amplifier (AC amplifier 1700, A-M Systems; or Brownlee Precision 440, Instrumentation Amplifier). Single neuron spikes were discriminated and digital pulses were collected online using a laboratory interface and software (CED1401, Spike2; Cambridge Electronic Design; or Datapac 2 K2, Run Technologies). The chemical manipulation of the tVTA was done on one cell only per animal. The electrical stimulation of the tVTA and the lesion study allowed multiple recordings from the same animal in which case recording tracks were done $100 \mu \mathrm{m}$ apart along the mediolateral and/or AP axes. At the end of the experi- 
ments, the recording electrode placement was marked by iontophoretic deposit of pontamine sky blue $(-20 \mu \mathrm{A}$, continuous current, 12-15 min). Electrical stimulation sites were marked by electrolytic lesion $(+20 \mu \mathrm{A}$, continuous current, $2 \mathrm{~min})$. Rats were deeply anesthetized with isoflurane (5\%) and decapitated. Brains were removed, snap-frozen in isopentane at $-80^{\circ} \mathrm{C}$, sectioned $(30 \mu \mathrm{m}$ coronal sections), mounted, and stained with neutral red to visualize recording, stimulation, and injection sites.

Electrical stimulation of the tVTA was conducted with a concentric bipolar electrode $(250 \mu \mathrm{m}$ diameter overall, $100 \mu \mathrm{m}$ diameter inner electrode that extended $100 \mu \mathrm{m}$ beyond the outer electrode; Phymep). Stimulations (100, $0.2-1.0 \mathrm{~mA}, 0.5 \mathrm{~Hz}, 0.5 \mathrm{~ms}$ duration pulses) were administered using a square pulse stimulator and stimulus isolator. In one set of experiments, we delivered single pulses of stimulation and in an independent experiment we delivered trains of three consecutive pulses of stimulations, separated by $50 \mathrm{~ms}$, at $1 \mathrm{~mA}$. Responses to electrical stimulation of the tVTA were evaluated and a peristimulus time histogram (PSTH) was generated online (Spike2 software) for each neuron.

An injection pipette $(30 \mu \mathrm{m}$ tip diameter) was used for chemical manipulation of tVTA activity, either stimulation by injecting $60 \mathrm{nl}$ of L-glutamate $(100 \mathrm{mM}$ in PBS) or inactivation by injecting $120 \mathrm{nl}$ of muscimol-BODIPY (0.8 $\mathrm{mM}$ in PBS).

\section{Rotometer}

Each animal was introduced into a cylindrical plastic chamber $(40 \mathrm{~cm}$ diameter, $46 \mathrm{~cm}$ high) placed under a camera. The number of contralateral and ipsilateral rotations was counted. Rats were first tested for baseline rotation, which allowed the exclusion of rats showing spontaneous rotation bias. The chosen time window (30 min) for maximal locomotor activity was between 25 and $55 \mathrm{~min}$ post injection. In a first set of experiments, rats underwent stereotaxic surgery for unilateral lesion of the tVTA, of the SNc or of the VTA. After 2 weeks of recovery, rats were tested in the rotometer following an acute injection of saline or amphetamine $(3 \mathrm{mg} / \mathrm{kg}$, i.p.). In a second set of experiments, we compared animals with unilateral partial lesion of the SNc to animals with concomitant ipsilateral lesion of the tVTA, the test was done 1 week after the surgeries. The lesions were visualized by using immunohistochemistry directed against $\mathrm{TH}$ or NeuN. The extent of the partial unilateral SNc lesion was evaluated by counting the number of SNc TH-positive cells on both sides of the brain, analyzing one section every $160 \mu \mathrm{m}$ over the full AP extent of the SNc.

\section{Rotarod}

Experiment 1. At least 2 weeks after bilateral lesion of the tVTA or the dorsolateral striatum, we assessed the overall rotarod performance (ORP) (Roto-Rod Series 8), a motor parameter shown to be affected in a rat model of Parkinsonism (Rozas et al, 1997). Rats were first trained at low speed (5 rotations per minute (r.p.m.)) to remain on the rod for $5 \mathrm{~min}$. Each fall was recorded and the animal immediately put back on the rotarod until the end of the 5 min training. On day 2, animals were tested at successively incremented rotation speeds with a 5 min cut off. ORP was calculated as the area under the curve for time against speed plot. On day 3, animals were tested with an accelerating speed ramp (0-45 r.p.m., 120 s). The locomotor activity was also measured during $2 \mathrm{~h}$ through a photocell system.

Experiment 2. A separate set of animals was used to assess the effect of bilateral tVTA lesion on motor skill learning. They were tested repeatedly on the rotarod under an accelerating speed ramp, allowing the measurement of increased performance over days ( $0-45$ r.p.m., 5 min cut off, 3 consecutive times per day, over 4 days). The daily values correspond to the average of the three consecutive daily exposures to the rotarod.

Experiment 3. To assess the effect of amphetamine on motor skill learning, rats were tested repeatedly for performance on an accelerating speed ramp (0-45 r.p.m., 180 s) over two days ( 3 sessions/day, 45 min between sessions). On the first day, a single injection of amphetamine was delivered immediately after the second session.

Experiment 4. The effect of amphetamine on motor performance was assessed in a separate group of animals. Rats were first overtrained with an accelerating speed ramp (0-45 r.p.m., 120 s) during 4 days, which allowed stable performances. On day 5 , the animals were tested $30 \mathrm{~min}$ after saline or amphetamine injection.

\section{Statistical Analysis}

Results are expressed as mean \pm SEM. Statistics are given in figure legends. We used Student's $t$-test or ANOVA to analyze the electrophysiological data. We performed LSD post hoc for the analysis of the SNc neuron evoked activity after electrical stimulation and Dunnet post hoc for the analysis of neuron activity after chemical manipulation. For the behavioral experiments, we used Student's $t$-test, test of comparison between the mean and a standard value (rotation experiments and tVTA vs DLS comparison), and ANOVAs followed by Duncan post hoc when applicable.

\section{RESULTS}

\section{The tVTA Neurons Project to the Nigrostriatal System}

Using tract tracing, a projection from the posterior end of the VTA to the SNc had been previously described (Ferreira et al, 2008). This SNc projecting region extends beyond the VTA per se, encompassing the newly described GABAergic tVTA (Jhou et al, 2009a; Kaufling et al, 2009, 2010a). Consistent with this view, we injected the anterograde tracers BDA (Supplementary Figure S1) or PhaL (Figure 1dg) into the tVTA, and highlighted a plexus of fibers in the SNc with appositions onto dopamine neurons and processes. To identify the specific link with the nigrostriatal pathway, we then delivered the retrograde tracer CTb into the dorsolateral striatum together with $\mathrm{BDA}$ into the tVTA. tVTA fibers displayed appositions onto striatumprojecting SNc neurons (Figure 1a-c). In order to evaluate whether the bouton contacts between tVTA fibers and dopamine neurons were actual synapses, we analyzed the 
a

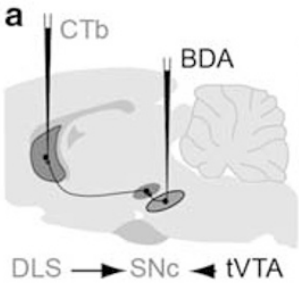

d

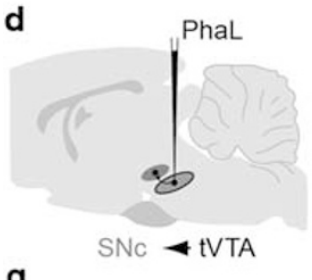

g

PhaL containing axons

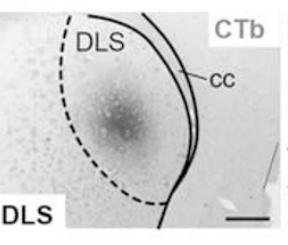

e

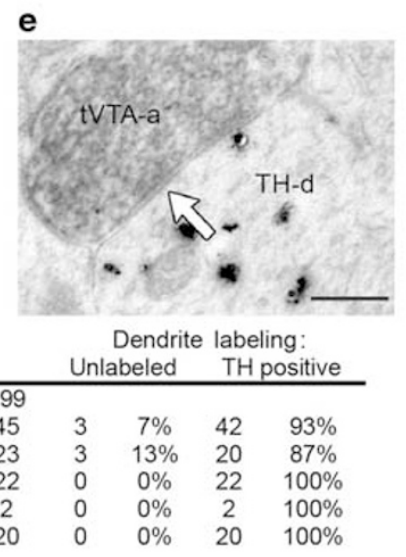

b

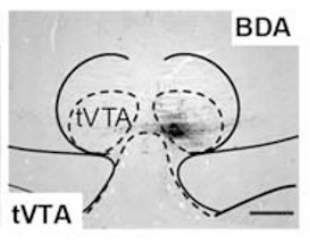

b

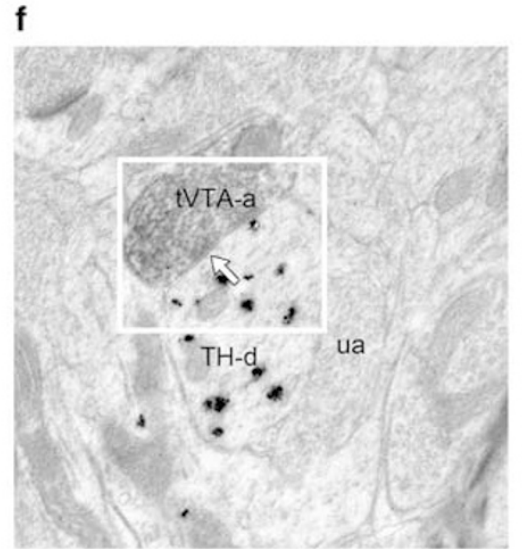

c

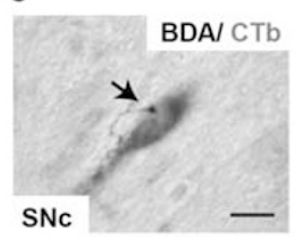

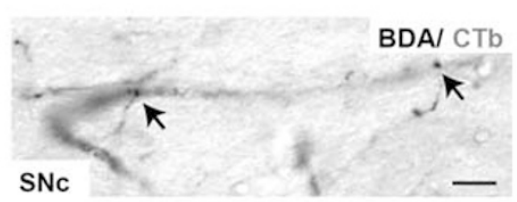

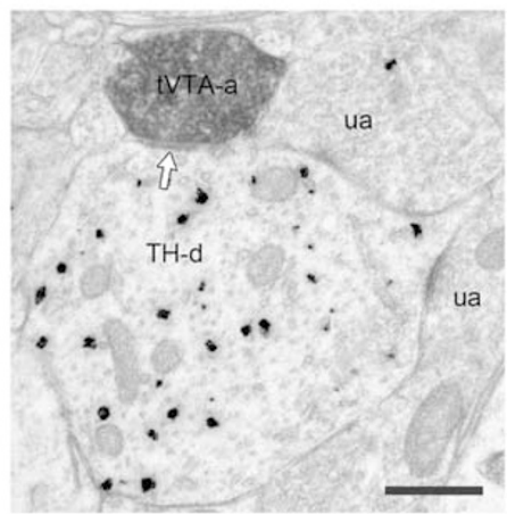

Figure I The tVTA neurons project to the nigrostriatal system. (a) Injection of the anterograde tracer BDA into the tVTA and of the retrograde tracer CTb into the dorsolateral striatum $(n=5)$. ( $b$ and c) tVTA terminals (arrows) contact dendrites and cell soma of striatum-projecting SNc neurons. (d) Injection of the anterograde tracer PhaL into the tVTA $(n=4)$. (e and f) Electron micrographs showing tVTA axon terminals (tVTA-a) forming synapses (arrow) onto SNc dendrites labeled for tyrosine hydroxylase (TH-d) and contacted by additional unlabeled axons (ua) ( $\mathrm{f}$ ), larger view; (e), details of boxed area). (g) Quantitative analysis of the electron microscopy experiment. Most of the tVTA terminals synapse onto dendrites in the SNc that are immunoreactive for TH. BDA, biotinylated dextran amine; cc, corpus callosum; CTb, cholera toxin $\beta$-subunit; DLS, dorsolateral striatum; PhaL, Phaseolus vulgaris leucoagglutinin. Scale bars, $500 \mu \mathrm{m}$ (a), $20 \mu \mathrm{m}$ (b, c), $0.25 \mu \mathrm{m}$ (e), $0.5 \mu \mathrm{m}$ (f).

SNc by electron microscopy after PhaL delivery into the tVTA. Most tVTA axons formed symmetric synapses with SNc dendrites immunoreactive for the dopamine synthetic enzyme TH (Figure 1d-g), showing that the tVTA-SNc projection is organized similarly to the tVTA-VTA projection (Balcita-Pedicino et al, 2011).

\section{The tVTA Controls the Activity of SNc Dopamine Neurons}

To examine the functional connectivity between the tVTA and the SNc, we then recorded in vivo the extracellular activity from single SNc dopamine neurons in anesthetized rats. Ninety-five percent of the recorded neurons were inhibited by a single pulse of electrical stimulation of the tVTA (Figure 2a-e and Supplementary Figure S2), with an onset latency for inhibition of $3.2 \pm 1.3 \mathrm{~ms}$ and a mean duration of $60.5 \pm 11.4 \mathrm{~ms}$. We also observed that the duration of inhibition of SNc dopamine neurons was inversely correlated with the basal firing rate, while the magnitude of the response was uncorrelated (Supplementary Figure S2). Using a train of three pulses of stimulation instead of one, we observed a more prolonged inhibition of SNc cells $(206.1 \pm 25.0 \mathrm{~ms}$; Supplementary Figure S2). The tVTA is, however, partially embedded within the fiber bundles of the superior cerebellar peduncle (Kaufling et al, 2009) and the dense passing fibers surrounding the tVTA could mediate part of the consequences of the electrical stimulation. Hence, microinjection of L-glutamate or a labeled $\mathrm{GABA}_{\mathrm{A}}$ receptor agonist, muscimol-BODIPY, was used to chemically stimulate or inhibit tVTA neurons, respectively, without affecting passing fibers. These mirror manipulations decreased (L-glutamate) or increased (muscimol-BODIPY) SNc dopamine neuron firing rate ( $-61 \pm 6 \%$ and $+113 \pm 43 \%$ of basal activity, respectively) and bursting rate $(-72 \pm 7 \%$ and $+132 \pm 42 \%$ of basal bursting rate, respectively) (Figure $2 \mathrm{f}-\mathrm{i}$ ), thus revealing that the tVTA exerts both phasic and tonic inhibitory controls on the activity of these cells.

\section{The tVTA Controls Motor Behavior}

The neuroanatomical and electrophysiological data were supportive of a main inhibitory control exerted by the tVTA onto nigrostriatal dopamine neurons, which raised the question of the behavioral significance of this influence. The rotation model following SNc unilateral lesion has been widely used in Parkinson's disease research, with lesions inducing ipsilateral rotations after amphetamine administration (Figure $3 \mathrm{c}$ and $\mathrm{d}$ ). This rotation bias is specific to the nigrostriatal pathway, as it is not present with VTArestricted lesions (Kelly and Moore, 1976) (Figure 3e and f). Unilateral excitotoxic lesion of the tVTA alone increased contralateral rotational behavior $(+25.4 \%)$ (Figure 3a and b), opposite to what was observed following unilateral $\mathrm{SNc}$ lesions (Figure 3d) and consistent with a dysregulation of the inhibitory control of SNc dopamine neurons. We then tested whether a lesion of the tVTA might compensate the 
b
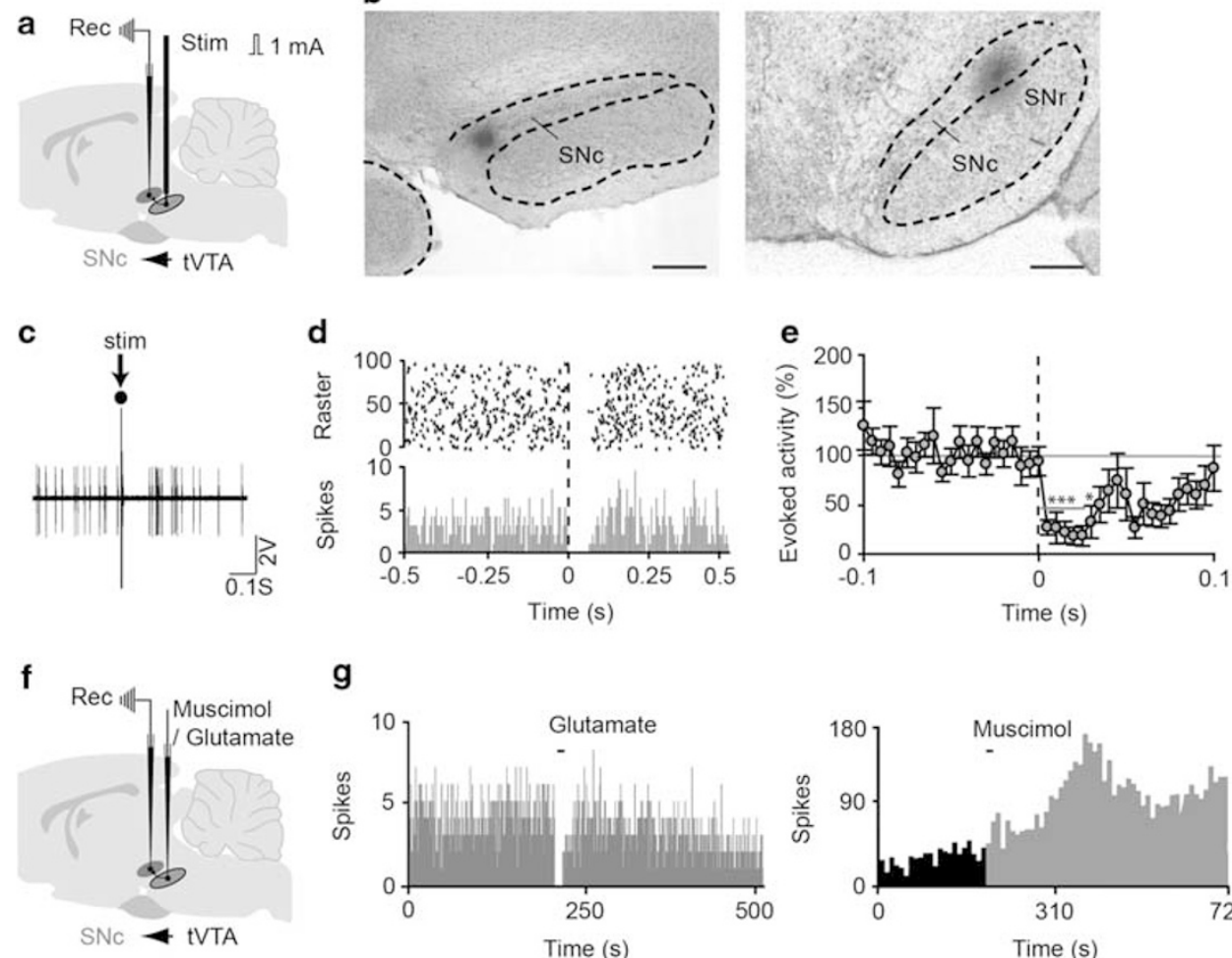

g
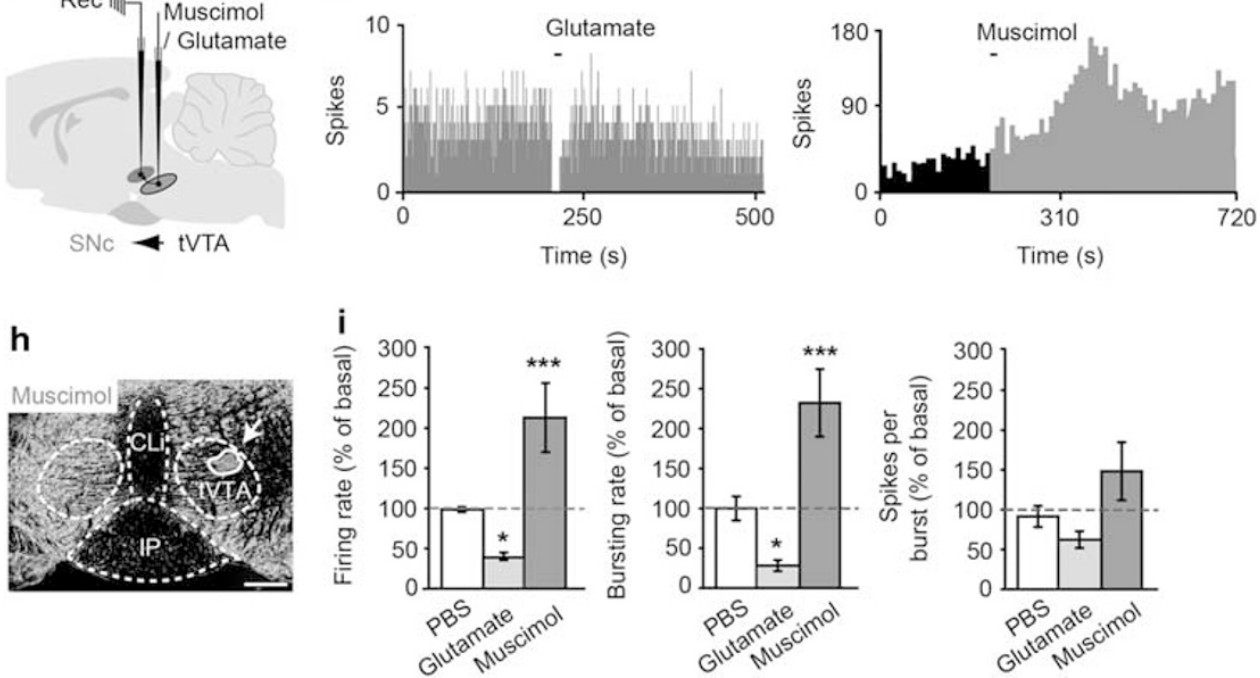

Figure 2 The tVTA controls SNc dopamine neuron activity. (a) Experimental protocol used for electrical stimulation. (b) Microphotographs of coronal sections through the SNc showing blue spots done at the end of recordings (left, anteromedial spot; right, posterolateral spot). (c) Example of a spike trace of a SNc dopamine neuron with a single pulse of tVTA stimulation at I mA. ( $d$ and e) Consequence of tVTA stimulation on a single SNc dopamine neuron (d) and on the mean of 19 neurons (e), $n=19, F_{40,720}=4.2, * p<0.05$, **** $p<0.00 \mathrm{I}$ ). ( $f$ ) Experimental protocol used for chemical stimulation and inhibition of the tVTA. (g) Examples of unitary activities from single SNc dopamine neurons after tVTA chemical manipulation. (h) Injection site for muscimol-BODIPY in the tVTA. (i) Consequences of tVTA inhibition (muscimol, $n=9$ ) or excitation (glutamate, $n=16$ ) on SNc dopamine neuron activity (vs PBS vehicle, $n=15$ ) (Firing rate $F_{2,37}=22.3, p<0.00$ I; bursting rate $F_{2,37}=23.6, p<0.00$ I; spikes/burst $F_{2,37}=4.9, p<0.02$; * $p<0.05$, **** $p<0.00$ I). Graphs represent mean \pm SEM. CLi, caudal linear nucleus of the raphe; IP, interpeduncular nucleus. Scale bar, $500 \mu \mathrm{m}$ (b and h).

rotation bias induced by a partial ipsilateral lesion of the SNc. The extent of the SNc lesion (Figure 3g) was similar in both groups $(53.9 \pm 7.1 \%$ vs $57.0 \pm 7.5 \%$ of SNc dopamine lesion for the SNc and the SNc-tVTA groups, respectively). While a strong ipsilateral amphetamine-induced rotation was observed after partial SNc lesion, this bias was absent in animals with concomitant lesion of the tVTA (Figure 3h).

To further explore nigrostriatal functions, we then studied motor performance. After bilateral tVTA lesion, we observed that the basal activity of SNc dopamine neurons was chronically enhanced (control, $3.5 \pm 0.3 \mathrm{~Hz}$; lesion, $5.2 \pm 0.5 \mathrm{~Hz}$ ) (Figure $4 \mathrm{~b}$ ), even though no significant locomotor hyperactivity was observed per se (Jhou et al, 2009b) (Figure 4c). Animals with ablation of the tVTA, however, displayed a major increase in motor performance in rotarod tasks. During habituation to the apparatus at low speed, tVTA-lesioned rats displayed fewer falls than control rats (control, $3.3 \pm 0.5$; lesion, $0.6 \pm 0.2$ ) (Figure $4 \mathrm{~d}$ ). After the habituation day, control rats had good performance at this low speed (Figure 4e), but a major difference with tVTA-lesioned rats was present at a more challenging fixed speed, with an improvement of ORP in bilateral tVTAlesioned rats compared with non-lesioned animals (ORP: control, $2262 \pm 149$; lesion, $4098 \pm 450$ ) (Figure 4e and f). The improvement also resulted in the capacity for animals without the tVTA to remain on the rotarod at higher speed than controls, as shown by the fall thresholds under a ramp acceleration procedure (r.p.m: control, $10.0 \pm 1.4$; lesion, 
a
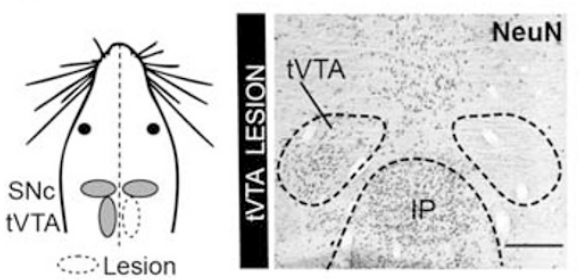

C
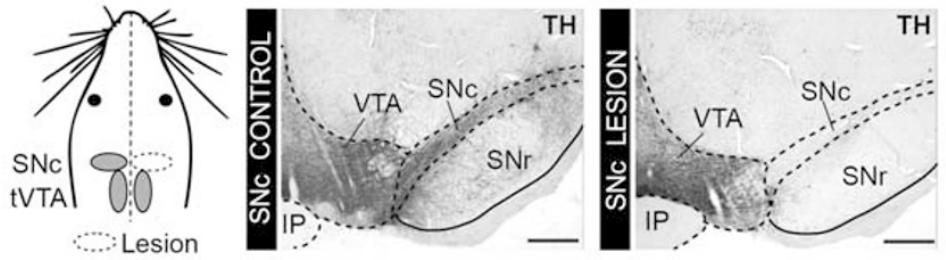

e
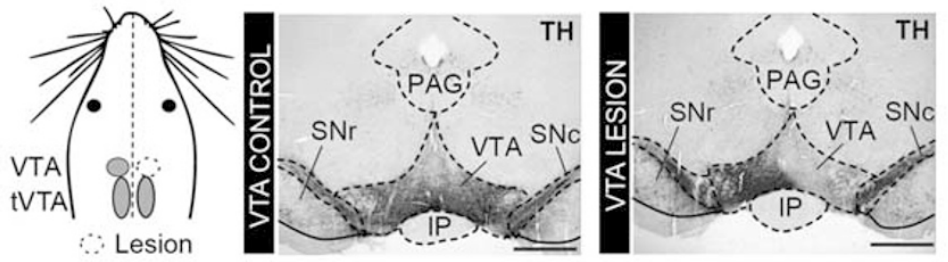
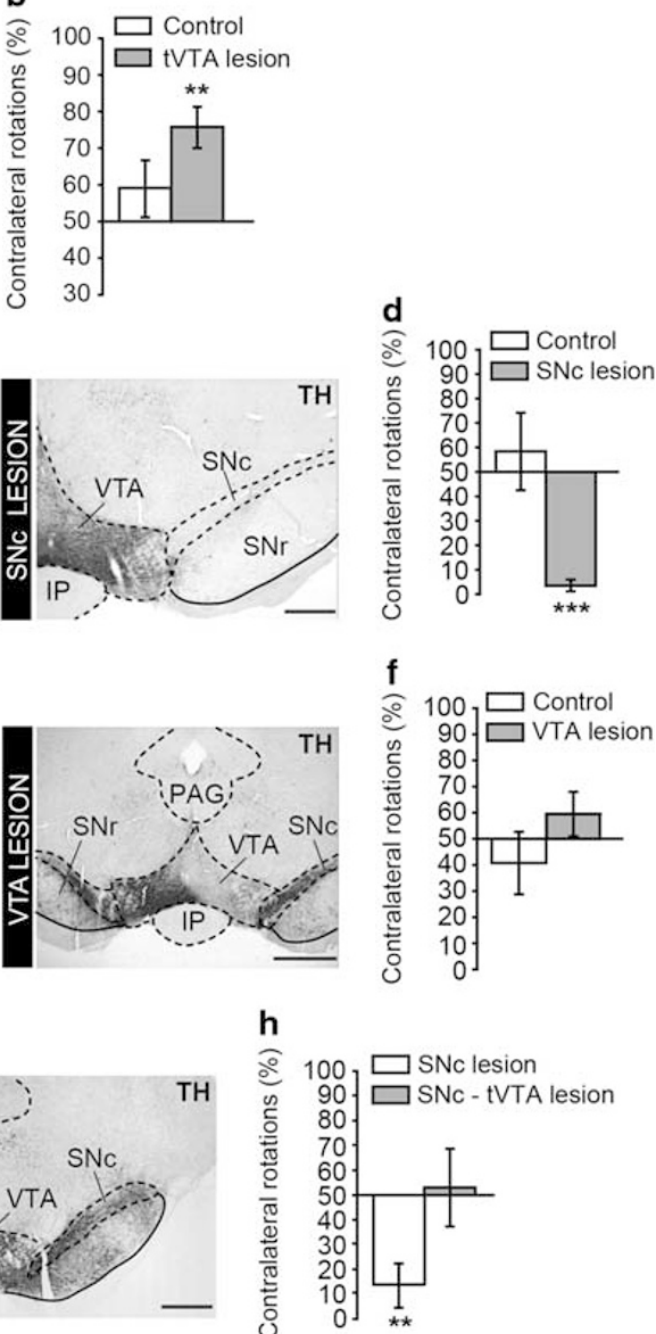

Figure 3 The tVTA controls rotation bias behavior. (a) Experimental protocol and histological evidence for unilateral tVTA lesion. (b) Unilateral tVTA lesion induces a contralateral rotation bias after amphetamine $3 \mathrm{mg} / \mathrm{kg}$ (control, $n=17$; lesion, $n=12$; $* * p<0.0 \mathrm{l}$ ). (c) Experimental protocol and histological evidence for 6-OHDA unilateral SNc lesion. (d) Unilateral SNc lesion induces an ipsilateral rotation bias after amphetamine administration (control, $n=12$; lesion, $n=14$; $*$ **** $p<0.000$ I referred to 50\%). (e) Experimental protocol and histological evidence for unilateral VTA lesion with ibotenic acid. (f) Unilateral VTA lesion does not induce a rotation bias after amphetamine administration (control, $n=9$; lesion, $n=7$; referred to 50\%). (g) Experimental protocol for unilateral SNc-tVTA lesions. The microphotograph illustrates a left side partial SNc lesion. (h) A tVTA lesion prevents the amphetamine-induced ipsilateral rotation bias observed after partial unilateral SNc lesion (SNc lesion, $n=8$; SNc-tVTA lesion, $n=7 ;$ *** $p<0.0$ l referred to 50\%). Graphs represent mean \pm SEM. 6-OHDA, 6-hydroxydopamine; IP, interpeduncular nucleus; PAG, periaqueductal gray; SNc, substantia nigra pars compacta; SNr, substantia nigra pars reticulata; TH, tyrosine hydroxylase; VTA, ventral tegmental area. Scale bars, $400 \mu \mathrm{m}$ (a), $500 \mu \mathrm{m}$ (c, e, g).

$18.5 \pm 1.9$ ) (Figure $4 \mathrm{~g}$ ). These performance enhancements following the tVTA ablation are opposite to the impairments observed following dopaminergic lesion of the dorsolateral striatum (Deumens et al, 2002) (Figure 5).

The motor improvement after bilateral tVTA lesion affected not only motor performance but also motor skill learning, as revealed in a specific experiment with repeated training over days (Figure $4 \mathrm{~h}$ ). In these conditions, tVTAlesioned animals increased their performance over days while a ceiling effect was present in controls (control, $135 \pm 22 \mathrm{~s}$ on day 3 ; lesion, $252 \pm 40 \mathrm{~s}$ on day 4). The increased nigrostriatal activity and associated motor performance following tVTA lesion somewhat parallel those of the 'performance enhancing' drug, amphetamine. Indeed, amphetamine enhances extracellular dopamine levels (Rebec, 2006; Sulzer, 2011), and in agreement with previous reports (Pryor et al, 1978), we observed that amphetamine increased motor performance in animals that had already learned the motor task (r.p.m: control, $13.9 \pm 1.6$; amphetamine, $21.2 \pm 2.5$ ) (Figure 6a). Interestingly, we also found that amphetamine can improve motor skill learning (Figure 6b). This action can be seen while under the acute influence of the drug (day 1 in Figure 6b), and the resulting improvement is long-lasting, persisting even though the animal is no more under the influence of the drug, as shown by the increased performance still present $24 \mathrm{~h}$ after the training done under amphetamine (day 2 in Figure $6 \mathrm{~b})$. 

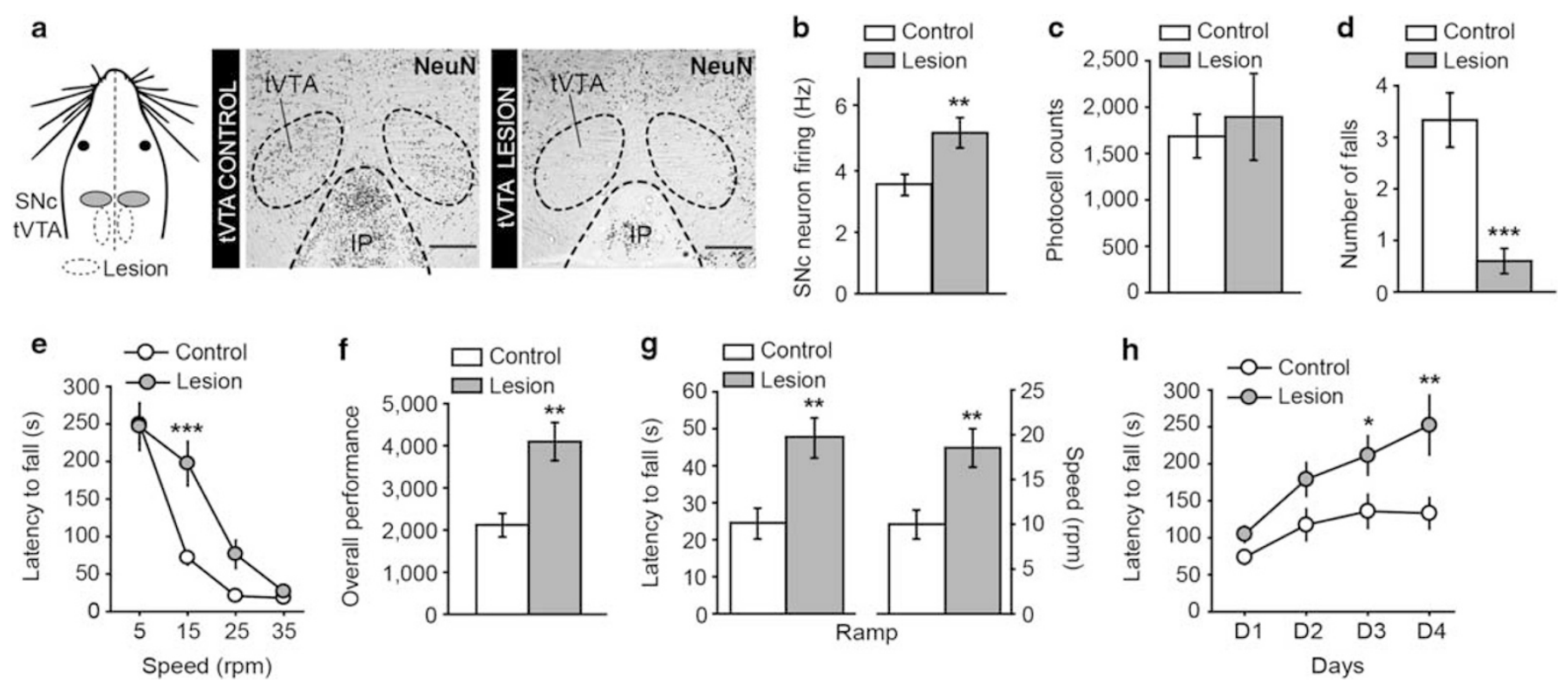

Figure 4 The tVTA controls motor behavior. (a) Experimental protocol and histological evidence for bilateral tVTA lesion. (b) Bilateral tVTA lesion increases SNc neuron basal firing (control, $n=22$; lesion, $n=23$; *** $<0.0$ I). (c) Locomotor activity of the bilateral tVTA-lesioned animals after subcutaneous saline injection (over $2 \mathrm{~h}$ ) (control, $n=9$; lesion, $n=10$ ). (d) Number of falls in bilateral tVTA-lesioned and non-lesioned animals during their first rotarod training at low speed ( 5 r.p.m., 300 s) (control, $n=9$; lesion, $n=10$; ***** $<0.00$ I). (e) Motor performance of the bilateral tVTA-lesioned animals in a rotarod task at successively increasing fixed speeds (control, $n=9$; lesion, $n=10 ; F_{3,5}=5.1, p<0.0 I$, ***** $p<0.00 \mathrm{I}$ ). (f) Overall performance comparison between bilateral tVTA-lesioned and non-lesioned animals, corresponding to e $(* * p<0.0 \mathrm{I})$. (g) Latency to fall and speed of bilateral tVTAlesioned animals in a ramp rotarod task (0-45 r.p.m. in I 20 s) (control, $n=9$; lesion, $n=10$; *** $p<0.0 \mathrm{I}$ ). (h) Motor skill learning of the bilateral tVTA-lesioned animals in a rotarod task measured over successive days (0-45 r.p.m., in 300 s) (control, $n=10$; lesion $=9$; $F_{3.51}=3.5, p<0.05$; * $p<0.05$, ** $p<0.0$ l). Graphs represent mean \pm SEM. r.p.m., rotations per minute; IP, interpeduncular nucleus. Scale bars, $500 \mu \mathrm{m}$ (a).
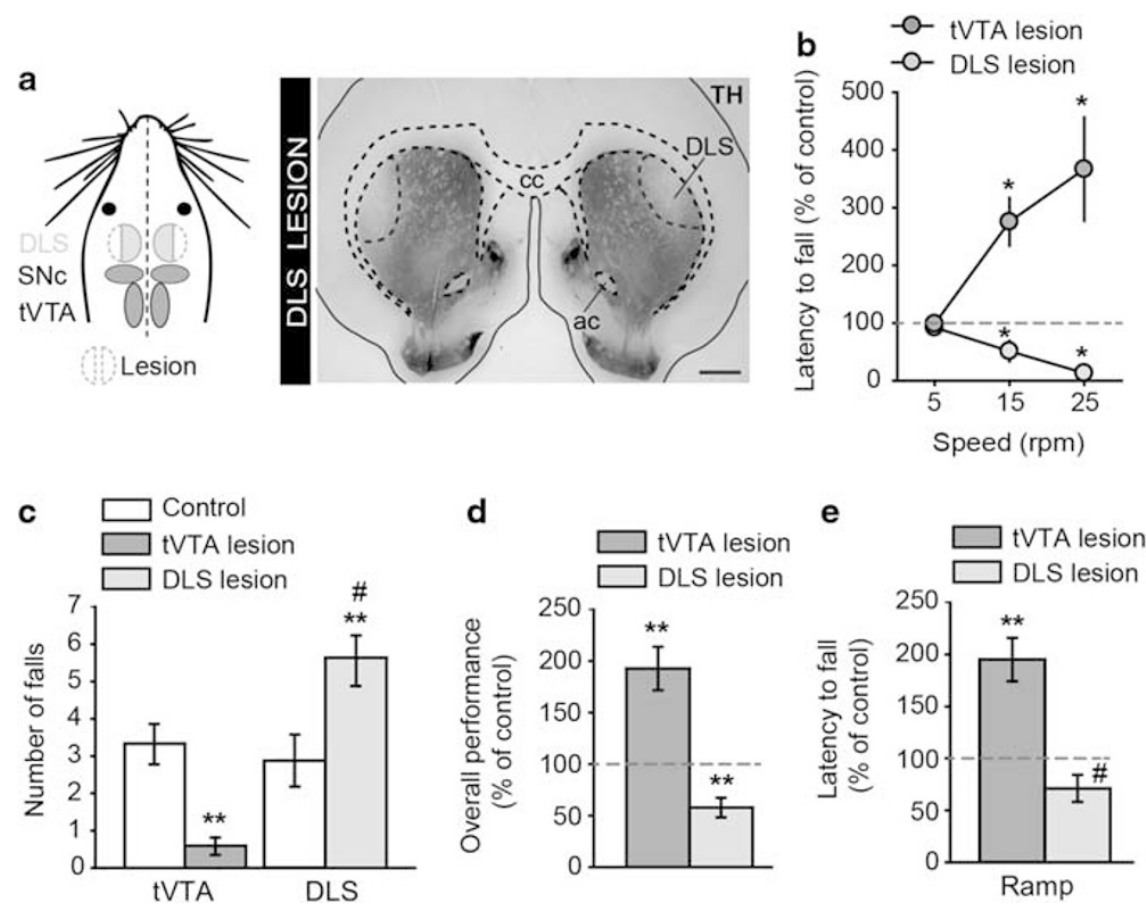

Figure 5 Comparison of motor performances between tVTA and dorsolateral striatum lesions. (a) Experimental protocol and histological evidence for bilateral DLS lesion with 6-OHDA. (b) Latency to fall of bilateral tVTA and DLS-lesioned animals in a rotarod task at fixed speed (tVTA, $n=10$, DLS, $n=1 \mathrm{I}$; referred to $100 \%, * p<0.05$. (c) Number of falls during the first day of rotarod training at low speed (control, $n=8-9 ;$ tVTA lesion, $n=10$; DLS lesion, $n=11$; $* * * 0.01$ compared with controls; tVTA-DLS comparison, ${ }^{\#} p<0.001$ ). (d) Overall performance comparison corresponding to Figure $5 b$

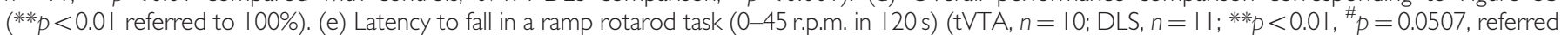
to $100 \%)$. Graphs represent mean \pm SEM. ac, anterior commissure; cc, corpus callosum; DLS, dorsolateral striatum; r.p.m., rotations per minute; TH, tyrosine hydroxylase. Scale bar, I mm (a). 

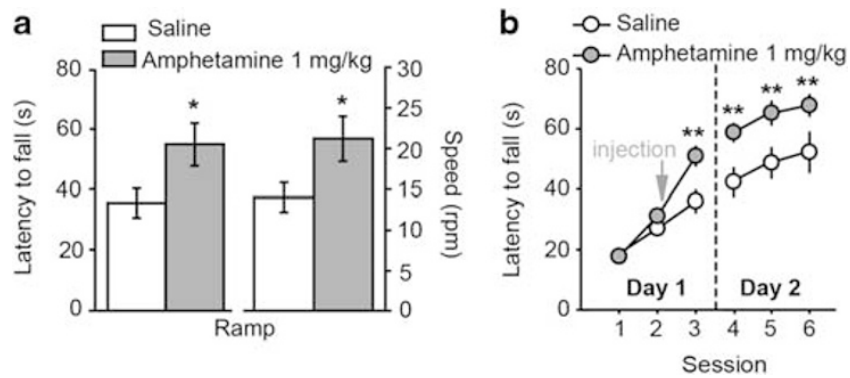

Figure 6 Amphetamine increases motor performance. (a) Amphetamine increases motor performance in a learned rotarod task (0-45 r.p.m., $120 \mathrm{~s}$ ) (saline, $n=10$; amphetamine, $n=10$; * $p<0.05$ ). (b) Amphetamine increases motor skill learning in a rotarod task assessed over successive days (0-45 r.p.m., $180 \mathrm{~s}$ ) (saline, $n=12$; amphetamine, $n=13$; $F_{5,115}=3.6$, $p<0.01$, *** $p<0.01)$. Graphs represent mean \pm SEM.

\section{DISCUSSION}

In this study, tract tracing and electron microscopy approaches allowed the visualization of a tVTA-nigrostriatal pathway. We then revealed the inhibitory influence of tVTA on the activity of SNc dopamine neurons by conducting in vivo electrophysiological studies. Together, these findings were supportive of a main role of the tVTA as a GABA brake for the nigrostriatal pathway. We then confirmed it at a behavioral level, first by observing an amphetamineinduced contralateral rotation bias following unilateral tVTA ablation, then by studying motor coordination and motor skill learning performances after bilateral ablation of the tVTA. The behavioral consequences of tVTA lesion are similar to the performance-enhancing action of a low dose of amphetamine, which also recruits dopamine systems. These findings highlight the major influence that the tVTA exerts over basal ganglia functions.

The anatomical data reveal a heavy projection from the tVTA to the nigrostriatal system. While studying the possible interconnections between the mesencephalic dopaminergic areas, a discrete subregion in the caudal pole of the VTA was previously shown to display efferents to the VTA-SNc complex (Ferreira et al, 2008). This subregion is now identified as the rostral part of the tVTA, a long GABA structure whose first tier is bilaterally inserted within the VTA and that spans rostrocaudally over $1 \mathrm{~mm}$ in rats, laterally to the median raphe nucleus and partially embedded with the superior cerebellar peduncle (Jhou et al, 2009a, 2009b; Kaufling et al, 2009, 2010a). Study of the tVTA-VTA efferents revealed that more than $80 \%$ of the synapses formed by tVTA axons in the VTA contacted the dendrites of dopamine cells (Balcita-Pedicino et al, 2011). The present data confirm the presence of a tVTA-SNc projection, and show appositions from tVTA fibers onto nigrostriatal neurons and the presence of symmetric synaptic connections between TVTA axons and TH-containing dendrites in the SNc. This structural organization is thus similar to the one that was observed in the VTA (Balcita-Pedicino et al, 2011), suggesting that the GABAergic tVTA should exert a main inhibitory control on both populations of dopamine cells.

Dopamine cells are under the control of excitatory and inhibitory inputs whose balance finely tunes cell activity
(Morikawa and Paladini, 2011). The tVTA is now recognized among the important inhibitory control regions for VTA cells (Barrot et al, 2012; Bourdy and Barrot, 2012; Jalabert et al, 2011; Lecca et al, 2011, 2012; Matsui and Williams, 2011), and the present data demonstrate that the tVTA is also critical for the regulation of SNc dopamine cell activity. Indeed, we show that the tVTA influences both the firing rate and bursting rate of SNc dopamine cells and that this control is both phasic and tonic. Single-pulse electrical stimulation of GABAergic inputs originating in the striatum, the globus pallidus or the substantia nigra pars reticulata produces an inhibition lasting around $30 \mathrm{~ms}$ (Paladini et al, 1999), whereas a similar stimulation in the tVTA produces an inhibition lasting around $60 \mathrm{~ms}$ (Figure 2c-e). These two independent sets of data collected in very similar experimental conditions highly suggest that the tVTA exerts a greater inhibition of the SNc dopamine neurons than the classical inhibitory inputs arising from the basal ganglia itself.

The presence of excitatory glutamate signaling can also be important to increase dopamine cell activity following the removal of the GABA brake on these neurons (Jalabert et al, 2011). In the case of SNc dopamine cells, such excitatory inputs are provided by structures within the basal ganglia circuitry but also from the somatosensory and from the primary and secondary motor cortices (Watabe-Uchida et al, 2012). This connectivity suggests that removing the tVTA braking influence could amplify the impact of these sensorimotor-related excitatory afferents on the nigrostriatal system whose stimulated state could then favor specific striatal functions.

Previous functional data on the tVTA concerned its influence on the mesolimbic system, more particularly in relation to aversive and avoidance behaviors (Jhou et al, 2009b; Stamatakis and Stuber, 2012) and to drugs of abuse (Jalabert et al, 2011; Jhou et al, 2012, 2013; Lecca et al, 2011, 2012; Matsui and Williams, 2011). Thus, GABA cells of the tVTA are targets for psychostimulants, including amphetamines, as shown by the expression of Fos proteins (Cornish et al, 2012; Jhou et al, 2009a, 2009b; Kaufling et al, 2009, 2010a, 2010b; Geisler et al, 2008; Lavezzi et al, 2011; Lecca et al, 2011; Matsui and Williams, 2011; Perroti et al, 2005; Rotllant et al, 2010; Scammel et al, 2000; Zahm et al, 2010). In rats, this expression is classically used to visualize the tVTA. Electrophysiologically, the tVTA neurons are also targets for cannabinoids and opiates (Jalabert et al, 2011; Lecca et al, 2011, 2012; Matsui and Williams, 2011). In the latter case, investigations of the tVTA recently led to an updated disinhibition model for opiate recruitment of VTA dopamine cells (Bourdy and Barrot, 2012; Jalabert et al, 2011; Johnson and North, 1992; Matsui and Williams, 2011) and behavioral evidence further supports the role of the tVTA in the rewarding and reinforcing properties of opiates (Jhou et al, 2012). Behaviorally, the tVTA has also been shown to be important for freezing in a fear conditioning paradigm or to a predator odor (Jhou et al, 2009b), and to promote active, passive, and conditioned avoidance behaviors when its inputs from the lateral habenula are stimulated (Stamatakis and Stuber, 2012). In monkeys, tVTA neurons can detect reward prediction errors (Hong et al, 2011). While progress has been made concerning the physiology of this brain region, behavioral studies have 
focused on reward/avoidance aspects. The neuroanatomical and electrophysiological data nevertheless suggest that the tVTA should also regulate nigrostriatal functions such as motor control.

Rotation bias and motor coordination performance are supportive of a main influence of the tVTA on nigrostriatal functions. The amphetamine-induced rotation bias following unilateral lesions is selective for the nigrostriatal pathway, as shown by the lack of such bias following unilateral VTA lesion (Kelly and Moore, 1976; present data); and the opposite bias observed with SNc and tVTA lesions is in agreement with an inhibitory influence of tVTA on the nigrostriatal pathway. The tVTA-lesion rotation bias is, however, milder than the SNc lesion one, which may be related to the connectivity of these regions and/or to amphetamine's properties. Indeed, the tVTA-SNc pathway also includes contralaterally directed fibers, although it is principally ipsilateral (Jhou et al, 2009a; Kaufling et al, 2010a), whereas the nigrostriatal pathway is more strictly lateralized. Moreover, amphetamine is able to directly release dopamine (Sulzer, 2011), an action that would be less affected by changes in dopamine neuron activity. Despite this mild effect of tVTA lesion alone, such a lesion can compensate for the strong rotation bias induced by a partial ipsilateral lesion of the SNc, which further highlights the control exerted by the tVTA on the nigrostriatal pathway.

Under mildly increased dopamine cell activity, as obtained by removing the tVTA GABA brake on SNc neurons, or after mildly enhanced aminergic transmission, as provided by a low dose of amphetamine, we observed an improvement of both motor performance and motorrelated learning. The execution of challenging coordinated movements requires a concerted action of motor and sensory systems, for which the cerebral cortex, the cerebellum, and the basal ganglia are crucial components (Groenewegen, 2003). The fine-tuning of these brain regions participates not only in the execution of movement but also in associated motor learning (Groenewegen, 2003; Penhune and Steele, 2012). Indeed, complex movements have to be learned and practiced repeatedly to improve their execution. Nigrostriatal dopamine is an important modulator of these mechanisms, which are impaired following the partial loss or disruption of this pathway (Akita et al, 2006; Beeler et al, 2010; Ogura et al, 2005; Willuhn and Steiner, 2008). In this context, plasticity at glutamatergic corticostriatal synapses within the dorsal striatum has been implicated in motor learning (Dang et al, 2006; Pisani et al, 2005; Yin et al, 2009) and is regulated by dopamine (Calabresi et al, 2007). Both $\mathrm{D}_{1}$ receptor-expressing striatonigral and $\mathrm{D}_{2}$ expressing striatopallidal mediumsized spiny neurons appear to be critical for optimal performance during early motor learning (Durieux et al, 2012). However, striatonigral neurons seem to be more crucial than striatopallidal neurons for longer-term gradual motor skill acquisition or for the execution of a previously learned motor sequence (Durieux et al, 2012), even though synaptic potentiation is preferentially expressed in striatopallidal neurons after extensive motor training (Yin et al, 2009). The tVTA, by setting the level of activity of dopamine systems, appears to be a major controller for basal ganglia functions. The fact that the lesion of the tVTA or a low dose of amphetamine lead to motor improvement suggests that these manipulations may operate within the physiological range and could thus favor a harmonious recruitment of the basal ganglia circuits and of the above-mentioned plasticity. In this respect, much higher doses of amphetamine are known to impair motor performance (Gerald and Gupta, 1977). Further studies will, however, be required to detail the mechanism leading to motor improvement after tVTA ablation, and in particular, its impact on the direct and indirect pathways and on striatal plasticity.

The neuroanatomical, electrophysiological and behavioral evidence supports a main role of the tVTA as a GABA brake for dopamine systems, and they raise the question of tVTA integration within basal ganglia circuitry (Albin et al, 1989; Redgrave et al, 2010; Tepper and Lee, 2007). Increasing nigrostriatal transmission in this circuit, either through tVTA suppression or an amphetamine-like performance enhancer, not only improves motor coordination but also motor skill learning, highlighting the learning influence of the dopamine system (Wise, 2004). These data warrant further functional research on the tVTA influence over dopamine-related functions in both physiological and dopamine-related pathological contexts.

\section{FUNDING AND DISCLOSURE}

This research was supported by the Centre National de la Recherche Scientifique, Université de Strasbourg, Université Bordeaux Segalen, Agence Nationale de la Recherche (ANR11-sv4-002), Fondation pour la Recherche Médicale, NIH (MH067937) and by The Michael J. Fox Foundation for Parkinson s Research. The authors declare no conflict of interest.

\section{ACKNOWLEDGEMENTS}

We thank Ms Dorothée Daniel for her technical support.

\section{REFERENCES}

Albin RL, Young AB, Penney JB (1989). The functional anatomy of basal ganglia disorders. Trends Neurosci 12: 366-375.

Akita H, Ogata M, Jitsuki S, Ogura T, Oh-Nishi A, Hoka S et al (2006). Nigral injection of antisense oligonucleotides to synaptotagmin I using HVJ-liposome vectors causes disruption of dopamine release in the striatum and impaired skill learning. Brain Res 1095: 178-189.

Balcita-Pedicino JJ, Omelchenko N, Bell R, Sesack SR (2011). The inhibitory influence of the lateral habenula on midbrain dopamine cells: ultrastructural evidence for indirect mediation via the rostromedial tegmental nucleus. J Comp Neurol 519: $1143-1164$.

Barrot M, Sesack SR, Georges F, Pistis M, Hong S, Jhou TC (2012). Braking dopamine systems: a new GABA master structure for mesolimbic and nigrostriatal functions. J Neurosci 32: 14094-14101.

Beeler JA, Cao ZF, Kheirbek MA, Ding Y, Koranda J, Murakami M et al (2010). Dopamine-dependent motor learning: insight into levodopa's long-duration response. Ann Neurol 67: 639-647.

Bourdy R, Barrot M (2012). A new control center for dopaminergic systems: pulling the VTA by the tail. Trends Neurosci 35: 681-690. 
Calabresi P, Picconi B, Tozzi A, Di Filippo M (2007). Dopaminemediated regulation of corticostriatal synaptic plasticity. Trends Neurosci 30: 211-219.

Cornish JL, Hunt GE, Robins L, McGregor IS (2012). Regional c-Fos and FosB/DeltaFosB expression associated with chronic methamphetamine self-administration and methamphetamineseeking behavior in rats. Neuroscience 206: 100-114.

Dang MT, Yokoi F, Yin HH, Lovinger DM, Wang Y et al (2006). Disrupted motor learning and long-term synaptic plasticity in mice lacking NMDAR1 in the striatum. Proc Natl Acad Sci USA 103: 15254-15259.

Deumens R, Blokland A, Prickaerts J (2002). Modeling Parkinson's disease in rats: an evaluation of 6-OHDA lesions of the nigrostriatal pathway. Exp Neurol 175: 303-317.

Durieux PF, Schiffmann SN, de Kerchove d'Exaerde A (2012). Differential regulation of motor control and response to dopaminergic drugs by $\mathrm{D} 1 \mathrm{R}$ and $\mathrm{D} 2 \mathrm{R}$ neurons in distinct dorsal striatum subregions. EMBO J 31: 640-653.

Ferreira JG, Del-Fava F, Hasue RH, Shammah-Lagnado SJ (2008). Organization of ventral tegmental area projections to the ventral tegmental area-nigral complex in the rat. Neuroscience 153: 196-213.

Geisler S, Marinelli M, Degarmo B, Becker ML, Freiman AJ, Beales $M$ et al (2008). Prominent activation of brainstem and pallidal afferents of the ventral tegmental area by cocaine. Neuropsychopharmacology 33: 2688-2700.

Gerald MC, Gupta TK (1977). The effects of amphetamine isomers on rotarod performance. Psychopharmacology 55: 83-86.

Groenewegen HJ (2003). The basal ganglia and motor control. Neural Plast 10: 107-120.

Hong S, Jhou TC, Smith M, Saleem KS, Hikosaka O (2011). Negative reward signals from the lateral habenula to dopamine neurons are mediated by rostromedial tegmental nucleus in primates. J Neurosci 31: 11457-11471.

Jalabert M, Bourdy R, Courtin J, Veinante P, Manzoni OJ, Barrot M et al (2011). Neuronal circuits underlying acute morphine action on dopamine neurons. Proc Natl Acad Sci USA 108: 16446-16450.

Johnson SW, North RA (1992). Opioids excite dopamine neurons by hyperpolarization of local interneurons. J Neurosci 12: 483-488.

Jhou TC, Geisler S, Marinelli M, Degarmo BA, Zahm DS (2009a). The mesopontine rostromedial tegmental nucleus: a structure targeted by the lateral habenula that projects to the ventral tegmental area of Tsai and substantia nigra compacta. J Comp Neurol 513: 566-596.

Jhou TC, Fields HL, Baxter MG, Saper CB, Holland PC (2009b). The rostromedial tegmental nucleus (RMTg), a GABAergic afferent to midbrain dopamine neurons, encodes aversive stimuli and inhibits motor responses. Neuron 61: 786-800.

Jhou TC, Xu SP, Lee MR, Gallen CL, Ikemoto S (2012). Mapping of reinforcing and analgesic effects of the mu opioid agonist Endomorphin-1 in the ventral midbrain of the rat. Psychopharmacology 224: 303-312.

Jhou TC, Good CH, Rowley CS, Xu SP, Wang H, Burnham NW et al (2013). Cocaine drives aversive conditioning via delayed activation of dopamine-responsive habenular and midbrain pathways. J Neurosci 33: 7501-7512.

Kaufling J, Veinante P, Pawlowski SA, Freund-Mercier MJ, Barrot M (2009). Afferents to the GABAergic tail of the ventral tegmental area in the rat. J Comp Neurol 513: 597-621.

Kaufling J, Veinante P, Pawlowski SA, Freund-Mercier MJ, Barrot M (2010a). Gamma-aminobutyric acid cells with cocaineinduced DeltaFosB in the ventral tegmental area innervate mesolimbic neurons. Biol Psychiatry 67: 88-92.

Kaufling J, Waltisperger E, Bourdy R, Valera A, Veinante P, Freund-Mercier MJ et al (2010b). Pharmacological recruitment of the GABAergic tail of the ventral tegmental area by acute drug exposure. Br J Pharmacol 161: 1677-1691.
Kelly PH, Moore KE (1976). Mesolimbic dopaminergic neurons in the rotational model of nigrostriatal function. Nature 263: 695-696.

Lavezzi HN, Parsley KP, Zahm DS, Lavezzi HN (2011). Mesopontine rostromedial tegmental nucleus neurons projecting to the dorsal raphe and pedunculopontine tegmental nucleus: psychostimulant-elicited Fos expression and collateralization. Brain Struct Funct 217: 719-734

Lecca S, Melis M, Luchicchi A, Ennas MG, Castelli MP, Muntoni AL et al (2011). Effects of drugs of abuse on putative rostromedial tegmental neurons, inhibitory afferents to midbrain dopamine cells. Neuropsychopharmacology 36: 589-602.

Lecca S, Melis M, Luchicchi A, Muntoni AL, Pistis M (2012). Inhibitory inputs from rostromedial tegmental neurons regulate spontaneous activity of midbrain dopamine cells and their responses to drugs of abuse. Neuropsychopharmacology 37: 1164-1176.

Matsui A, Williams JT (2011). Opioid-sensitive GABA inputs from rostromedial tegmental nucleus synapse onto midbrain dopamine neurons. J Neurosci 31: 17729-17735.

Morikawa H, Paladini CA (2011). Dynamic regulation of midbrain dopamine neuron activity: intrinsic, synaptic, and plasticity mechanisms. Neuroscience 198: 95-111.

Ogura T, Ogata M, Akita H, Jitsuki S, Akiba L, Noda K et al (2005). Impaired acquisition of skilled behavior in rotarod task by moderate depletion of striatal dopamine in a pre-symptomatic stage model of Parkinson's disease. Neurosci Res 51: 299-308.

Omelchenko N, Bell R, Sesack SR (2009). Lateral habenula projections to dopamine and GABA neurons in the rat ventral tegmental area. Eur J Neurosci 30: 1239-1250.

Paladini CA, Celada P, Tepper JM (1999). Striatal, pallidal, and pars reticulata evoked inhibition of nigrostriatal dopaminergic neurons is mediated by GABA(A) receptors in vivo. Neuroscience 89: 799-812.

Paxinos G, Watson C (2007). The Rat Brain In Stereotaxic Coordinates. 4th edn. Academic: San Diego, CA, USA.

Penhune VB, Steele CJ (2012). Parallel contributions of cerebellar, striatal and M1 mechanisms to motor sequence learning. Behav Brain Res 226: 579-591.

Perrotti LI, Bolaños CA, Choi KH, Russo SJ, Edwards S, Ulery PG et al (2005). DeltaFosB accumulates in a GABAergic cell population in the posterior tail of the ventral tegmental area after psychostimulant treatment. Eur J Neurosci 21: 2817-2824.

Pisani A, Centonze D, Bernardi G, Calabresi P (2005). Striatal synaptic plasticity: implications for motor learning and Parkinson's disease. Mov Disord 20: 395-402.

Pryor GT, Larsen FF, Husain S (1978). Interactions of delta9tetrahydrocannabinol with d-amphetamine, cocaine and nicotine in rats. Pharmacol Biochem Behav 8: 295-318.

Rebec GV (2006). Behavioral electrophysiology of psychostimulants. Neuropsychopharmacology 31: 2341-2348.

Redgrave P, Rodriguez M, Smith Y, Rodriguez-Oroz MC, Lehericy $\mathrm{S}$, Bergman $\mathrm{H}$ et al (2010). Goal-directed and habitual control in the basal ganglia: implications for Parkinson's disease. Nat Rev Neurosci 11: 760-772.

Rotllant D, Márquez C, Nadal R, Armario A (2010). The brain pattern of c-fos induction by two doses of amphetamine suggests different brain processing pathways and minor contribution of behavioural traits. Neuroscience 168: 691-705.

Rozas G, Guerra MJ, Labandeira-Garcia JL (1997). An automated rotarod method for quantitative drug-free evaluation of overall motor deficits in rat models of parkinsonism. Brain Res Prot 2: $75-84$.

Scammell TE, Estabrooke IV, McCarthy MT, Chemelli RM, Yanagisawa M, Miller MS et al (2000). Hypothalamic arousal regions are activated during modafinil-induced wakefulness. J Neurosci 20: 8620-8628. 
Stamatakis AM, Stuber GD (2012). Activation of lateral habenula inputs to the ventral midbrain promotes behavioral avoidance. Nat Neurosci 15: 1105-1107.

Sulzer D (2011). How addictive drugs disrupt presynaptic dopamine neurotransmission. Neuron 69: 628-649.

Tepper JM, Lee CR (2007). GABAergic control of substantia nigra dopaminergic neurons. Prog Brain Res 160: 189-208.

Ungless MA, Magill PJ, Bolam JP (2004). Uniform inhibition of dopamine neurons in the ventral tegmental area by aversive stimuli. Science 303: 2040-2042.

Watabe-Uchida M, Zhu L, Ogawa SK, Vamanrao A, Uchida N (2012). Whole-brain mapping of direct inputs to midbrain dopamine neurons. Neuron 74: 858-873.
Willuhn I, Steiner H (2008). Motor-skill learning in a novel running-wheel task is dependent on D1 dopamine receptors in the striatum. Neuroscience 153: 249-258.

Wise RA (2004). Dopamine, learning and motivation. Nat Rev Neurosci 5: 483-494.

Yin HH, Mulcare SP, Hilário MR, Clouse E, Holloway T, Davis MI et al (2009). Dynamic reorganization of striatal circuits during the acquisition and consolidation of a skill. Nat Neurosci 12: 333-341.

Zahm DS, Becker ML, Freiman AJ, Strauch S, Degarmo B, Geisler S et al (2010). Fos after single and repeated self-administration of cocaine and saline in the rat: emphasis on the Basal forebrain and recalibration of expression. Neuropsychopharmacology 35: 445-463.

Supplementary Information accompanies the paper on the Neuropsychopharmacology website (http://www.nature.com/npp) 